lateral nephrectomy. ${ }^{5}$ To show this decisively, future studies should quantitate the amount of thyroid-binding globulin in pleural fluid.

\section{Conclusion}

When chest tube drainage leads to loss of Ig and ATIII, it is important to document that thyroid function is normal. Although the mechanism by which hypothyroidism occurs is not conclusively proven, thyroid hormone replacement may be necessary in instances in which prolonged chest tube drainage occurs.

\section{References}

1. Kelley RE. Stroke in the postoperative period. Med Clin North Am. 2001:85:1263-76.

2. McBride ME. Hypogammaglobulinemia complicating chylothorax after cardiac surgery in two infants. J Cardiothorac Vasc Anesth. 2001;15:358-61.

3. Ross OC, Petros A. The sick euthyroid syndrome in paediatric cardiac surgery patients. Intensive Care Med. 2001;27:1124-32.

4. Rondanini GF, de Panizza G, Bollati A, Manzoni P, Terenghi A, Mutinelli MR, et al. Congenital hypothyroidism and pericardial effusion. Horm Res. 1991;35:41-4.

5. Chadha V, Alon US. Bilateral nephrectomy reverses hypothyroidism in congenital nephritic syndrome. Pediatr Nephrol. 1999;13:209-11.

\title{
Stage III empyema caused by Actinomyces meyeri: A plea for decortication
}

\author{
Cédric Vallet, ${ }^{\mathrm{a}}$ Edgardo Pezzetta, ${ }^{\mathrm{a}}$ Geneviève Nicolet-Chatelin, ${ }^{\mathrm{b}}$ Zihad El Lamaa, \\ Olivier Martinet, ${ }^{a}$ and Hans-Beat Ris, ${ }^{a}$ Lausanne, Switzerland
}

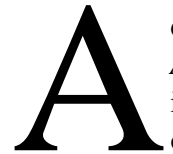
ctinomycosis is a rare infectious disease caused by Actinomyces species, a genus of anaerobic gram-positive bacillus. Actinomyces organisms are saprophytes of the human digestive tract and are very sensitive to penicillin. Thoracic actinomycosis represents $15 \%$ to $20 \%$ of cas$\mathrm{es}^{1-4}$ with pleural effusion in only $20 \%$ of intrathoracic affection. ${ }^{1,4,5}$ The usual clinical picture is that of a pleural effusion or pleural empyema together with a parenchymatous infection. We report a case of pleural effusion caused by Actinomyces meyeri without pulmonary involvement that failed to respond to chest tube drainage and antibiotherapy.

\section{Clinical Summary}

A 64-year-old woman was admitted with a history of fever and a dry cough associated with asthenia, weight loss, and exertional dyspnea. There was no previous medical history except for smoking and alcohol abuse. Laboratory studies showed a hemoglobin value of $9.9 \mathrm{~g} / 100 \mathrm{~mL}$, a hematocrit value of $25 \%$, a leukocyte count of $13,200 / \mathrm{mm}^{3}$, and a sedimentation rate of greater than 120 $\mathrm{mm} / \mathrm{h}$. Hemocultures were sterile, and the result of an HIV test was

From the Departments of Thoracic and Vascular Surgery a and Pulmonary Medicine, ${ }^{\text {b }}$ University Hospital, Lausanne, Switzerland.

Received for publication Oct 7, 2003; accepted for publication Nov 4, 2003.

Address for reprints: Cédric Vallet, Department of Thoracic and Vascular Surgery, University Hospital, Rue du Bugnon 46, CH-1011 Lausanne, Switzerland (E-mail: cvallet@hospvd.ch).

J Thorac Cardiovasc Surg 2004;127:1511-3

$0022-5223 / \$ 30.00$

Copyright $\odot 2004$ by The American Association for Thoracic Surgery

doi:10.1016/j.jtcvs.2003.11.043 negative. A chest radiograph showed a massive left pleural effusion (Figure 1). Chest tube drainage was performed, and this revealed a purulent effusion. A meyeri was identified after culture of the pleu-

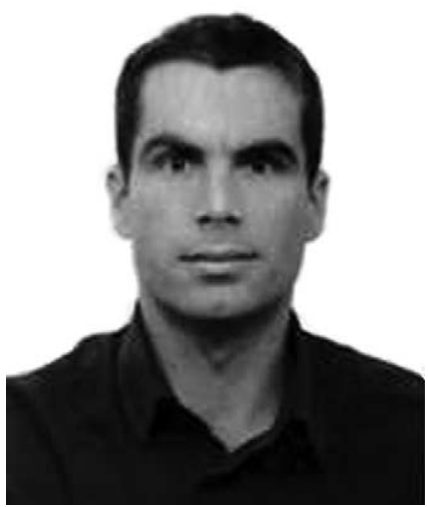

Dr Vallet ral effusion. Despite treatment with intravenous penicillin (20 Mio IU/d), a loculated pleural effusion developed. Chest computed tomography (CT) demonstrated thickening and enhancement of the parietal pleura and confirmed loculation of pleural effusion and left lower lobe atelectasis (Figure 2). Bronchial carcinoma was excluded by means of bronchoscopy and transbronchial biopsy of the left lower lobe. Pulmonary function testing revealed moderated obstruction and an important restriction, with a forced expiratory volume in 1 second of $1.04 \mathrm{~L}$ (47\% of predicted value), a forced vital capacity of 1.72 $\mathrm{L}(66 \%$ of predicted value), and a total lung capacity of $3.58 \mathrm{~L}$ ( $72 \%$ of predicted value). The patient remained febrile without clinical improvement and complained of pain at the site of previous drainage. A stage III empyema was diagnosed, and an operation was performed.

At thoracotomy, we found a pleurosubcutaneous fistula at the site of thoracic drainage mimicking an empyema necessitatis. The parietal and visceral pleura were thickened and adherent to adjacent structures, as usually seen in advanced mesothelioma. Decortication was performed with resection of the sinus tract, including the sixth rib and its interspace. Microbiologic examination of the pleura was performed, and $A$ meyeri was found in the pleural loculations filled with putrid liquid. The patient presented no postoperative complication and was discharged after 16 days. An oral penicillin regimen was prescribed for 6 months. Clinical and radiologic evolutions were favorable, with minimal pleural sequellae on chest radiography. Functional evaluation at 6 months after the operation revealed a forced expiratory volume in 1 second of 


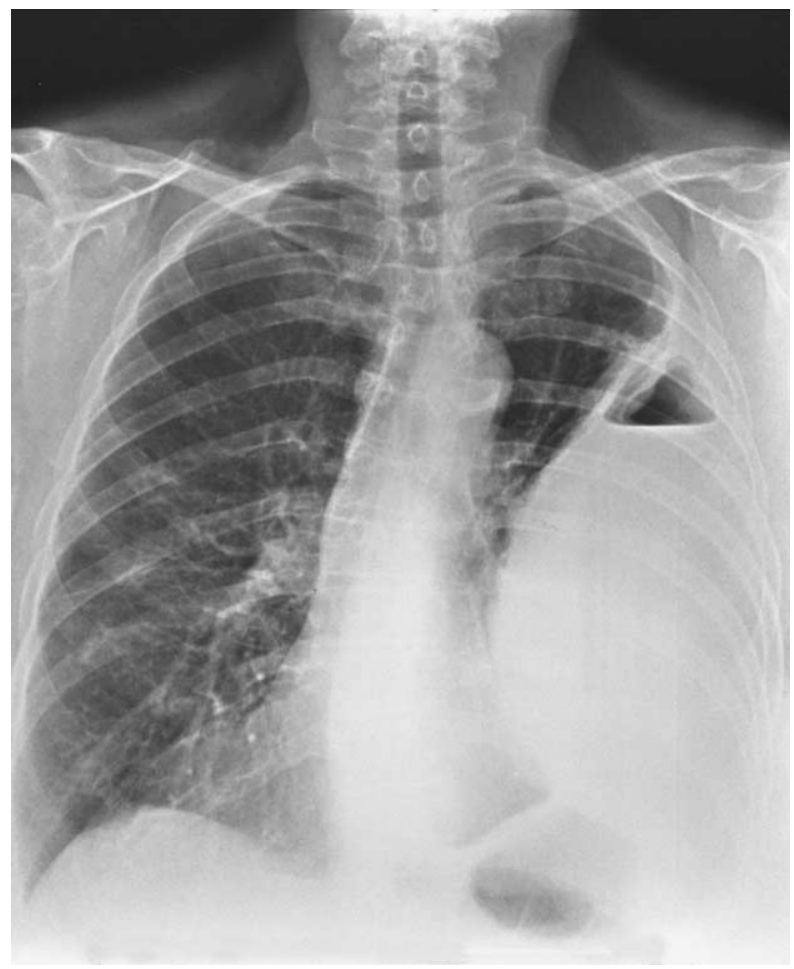

Figure 1. Chest radiograph showing pleural effusion related to $A$ meyeri.

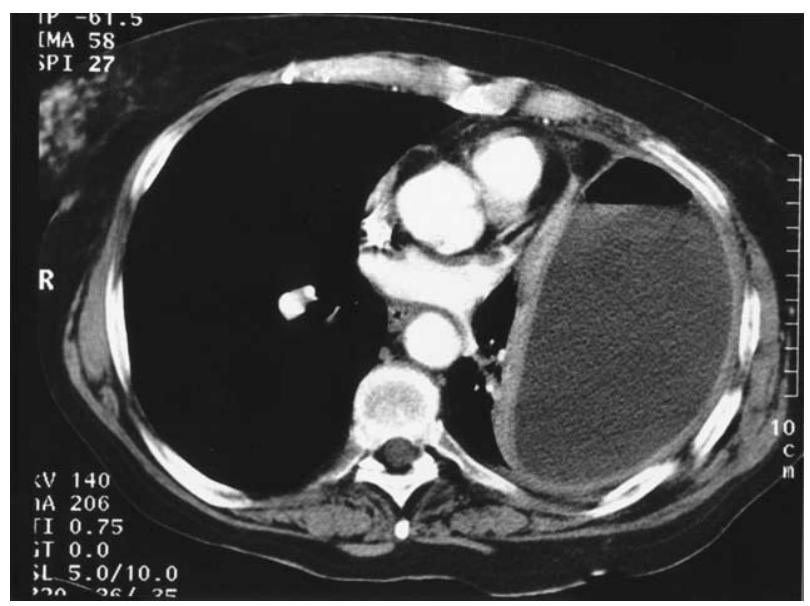

Figure 2. Chest CT scan showing loculated effusion, pleural enhancement, and atelectasis of the left lower lobe without signs of parenchymal actinomycosis.

$1.49 \mathrm{~L}$ ( $68 \%$ of predicted value) and a forced vital capacity of 2.52 L (96\% of predicted value). The patient gained weight, and thoracic pain disappeared. Fiberoptic bronchoscopy 7 months after the operation revealed a benign fibroepithelial polyp at the origin of the lower lobe bronchus that was resected with biopsy forceps. Bacteriologic cultures of bronchial aspiration remained sterile.

\section{Discussion}

The genus Actinomyces consists of gram-positive, non-acid-fast, nonmotile organisms that never produce spores and usually require an anaerobic environment for growth. Actinomyces organisms are considered bacteria because of their cell-wall component and susceptibility to antibacterial drugs, such as penicillin. ${ }^{6}$ Actinomycosis is a chronic, suppurative granulomatous infection. It typically involves cervicofacial and abdominopelvic organs. Thoracic involvement is less frequent.

Actinomyces species are commensals of the human oropharynx, gastrointestinal tract, and female genitalia and are often routinely cultured from these mucosa-lined orifices. Fourteen species have been characterized to date. ${ }^{7}$ Six of these are thought to be pathogenic in human subjects, including Actinomyces israelii, Actinomyces neslundi, Actinomyces odontolyticus, Actinomyces viscosus, A meyeri, and Actinomyces gerencseriae, and $A$ israelli is the organism most commonly incriminated in human disease. In contrast to other species, A meyeri might have a greater tendency of affecting the lung and hematogenous dissemination. A meyeri is an obligatory anaerobe organism that can be distinguished from other Actinomyces species on the basis of colony morphologic aspects, biochemical tests, acid production, end products from sugars, and cell-wall peptidoglycan analysis. ${ }^{6}$ These microorganisms might become pathogenic in debilitated patients who abuse alcohol and have bad oral hygiene. Infection is prevalent in men aged 30 to 50 years. $^{2,3,8}$

A crucial step in the development of clinical manifestation of the disease is the disruption of the mucosal barrier, allowing the organisms to invade. Pulmonary infection usually occurs after bronchial aspiration of oropharyngeal or gastrointestinal contents and rarely after an esophageal injury or by means of hematogenous spread. Pleural actinomycosis starts as a bronchopulmonary disease and involves the pleura through lymphatic spread. ${ }^{9}$ Aspiration of saliva or other material containing Actinomyces species might then cause atelectasis and pneumonitis. The initial acute inflammation is followed by the characteristic chronic and indolent phase of the disease that generates abscesses, necrosis, and fibrosis of lung parenchyma, which commonly cavitates. It progresses slowly without respecting anatomic boundaries, such as interlobar fissures or the chest wall. If not recognized, the parenchymal disease invades the pleura, the chest wall, soft tissues, and bony structures. Sinus tract formation and empyema necessitatis might arise, causing purulent discharge with yellowish sulfur granules. ${ }^{10}$ In our patient the sinus tract followed the chest tube into the subcutaneous tissue at the time of the operation, with imminent fistulization of the skin.

Clinical features are nonspecific and similar to those of other chronic suppurative chest diseases. Physical signs are equally nonspecific except in advanced and untreated disease, during which fistulization might be seen.

Imaging is useful in evaluating the extent and chronicity of disease. Definitive diagnosis is confirmed by means of histologic or microbiologic examination confirmation. Plain chest radiograph findings in actinomycosis are nonspecific. CT is more helpful in providing information on parenchymal involvement and extension into the adjacent chest wall. Pleural effusion with or without 
pleural thickening might also be diagnosed on the basis of CT scans.

Compared with pulmonary actinomycosis, the bacteriologic diagnosis is easily obtained by means of thoracocentesis in case of pleural involvement. However, diagnosis might be obscured by previous antibiotic therapy and a lack of anaerobic conditions for culture. At direct microbiologic examination, actinomycosis has to be suspected if gram-positive filamentous germs are discovered. An anaerobic enriched environment is needed for appropriate culture.

Penicillin G still remains the drug of choice, and the mainstay of treatment is the administration of high-dose intravenous penicillin. Eighteen to 24 millions units of penicillin per day are given for 2 to 6 weeks, followed by oral penicillin $\mathrm{V}$ administration for another 6 to 12 months. The evolution should be monitored by use of plain radiographs or $\mathrm{CT}$.

In stage I empyema, indication for surgical intervention is uncommon, but the efficacy of antibiotic therapy depends on the onset of diagnosis. If the treatment is delayed, stage II and III empyemas can develop, and decortication is required. Chest-wall fistulization has to be resected during the intervention. A VATS approach is usually unrewarding because of the chronicity of empyema at the time of the operation. After the operation, penicillin administration is recommended.

\section{References}

1. Bates M. Thoracic actinomycosis. Thorax. 1957;12:99-124.

2. Benhoff D. Actinomycosis: diagnostic and therapeutic considerations and review of 32 cases. Laryngoscope. 1984;94:1198-217.

3. Brown JR. Human actinomycosis. A study of 181 subjects. Hum Pathol. 1973;4:319-30.

4. Mabeza G, Macfarlane J. Pulmonary actinomycosis. Eur Respir J. 2003;21:545-51.

5. Hsieh MJ, Liu HP, Chang JP, Chang CH. Thoracic actinomycosis. Chest. 1993;104:366-70.

6. Rose HD, Varkey B, Kutty CP. Thoracic actinomycosis caused by Actinomyces meyeri. Am Rev Respir Dis. 1982;125:251-4.

7. Smego R, Foglia G. Actinomycosis. Clin Infect Dis. 1998;26:1255-63.

8. Weese W, Smith I. A study of 57 cases of actinomycosis over a 36 year period. Arch Intern Med. 1975;135:562-8.

9. Touboul JL, Chaussade F, Desvignes B, Mangeney T, Bidault C. Isolated pleural actinomycosis. A propos of 2 cases. Rev Pneumol Clin. 1990;46:69-72.

10. Hooker TP, Hammond M, Corral K. Empyema necessitatis: review of the manifestations of thoracic actinomycosis. Cleve Clin J Med. 1992; 59:542-8.

\title{
Thoracic endometriosis: A case report and literature review
}

\author{
Tomasz M. Ziedalski, MD, ${ }^{a, b}$ Viji Sankaranarayanan, MD, ${ }^{a, b}$ and Rajinder K. Chitkara, MD, ${ }^{a, b}$ Palo Alto, Calif
}

$\mathrm{T}$ horacic endometriosis is a rare disorder characterized by the presence of functioning endometrial tissue within the pleura, the lung parenchyma, or the airways. In this report we describe a case of catamenial hemothorax and review the various presentations, pathogenesis, diagnosis, and therapies of thoracic endometriosis.

\section{Clinical Summary}

A 28-year-old woman was seen for cough, right-sided pleuritic chest pain, and dyspnea. During the previous 3 years she had had multiple hospitalizations for right-sided pneumothorax, hemo-

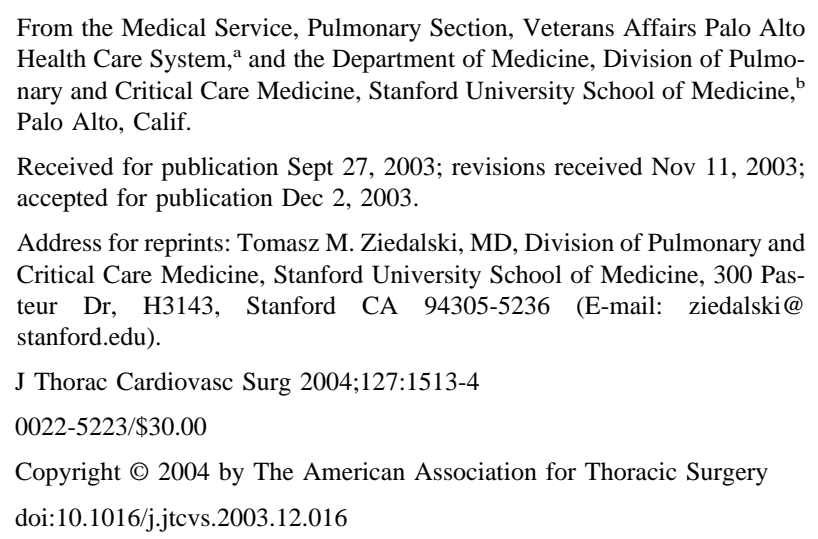

pneumothorax, and hemothorax. She had negative results of a workup for pulmonary embolism and of a pleural biopsy. The symptoms occurred within 24 to 48 hours after the onset of menses. Danazol therapy was unsuccessful and she refused hysterectomy with oophorectomy. Examination revealed a pale, mildly dyspneic woman with decreased breath sounds at the right base of the lungs. Chest radiography demonstrated a right-sided air-fluid level. Thoracentesis drained hemorrhagic fluid. Culture results were negative, and cytologic examination demonstrated endometrial epithelial cells with no evidence of malignancy. The postthoracentesis chest radiograph showed a small rounded pleural density and gas-liquid level on the right side. The patient subsequently underwent pleurectomy, with removal of the pleural implant (Figures 1 and 2) and talc pleurodesis. One year later, she was free of symptoms.

\section{Discussion}

Thoracic endometriosis is a rare disorder with varying clinical presentations. The largest series reviewed 112 cases of thoracic pathology associated with menstruation presenting as hemoptysis, hemothorax, pneumothorax, or pulmonary nodules. ${ }^{1}$ The term thoracic endometriosis syndrome (TES) was proposed. The mean age at diagnosis was 35 years, with a range from 19 to 54 years. Pneumothorax occurred in $73 \%$ of the cases, hemothorax in $14 \%$, hemoptysis in 7\%, and nodules in 6\%. Patients had chest pain in $90 \%$ of the cases and dyspnea in $31 \%$. In $90 \%$ of patients, the symptoms occurred within 2 days after the onset of menstruation. 